\title{
Beam-ACO for the Longest Common Subsequence Problem
}

\author{
Christian Blum
}

\begin{abstract}
The longest common subsequence problem is classical string problem. It has applications, for example, in pattern recognition and bioinformatics. In this work we present a socalled Beam-ACO approach for solving this problem. BeamACO algorithms are hybrid techniques that results from a combination of ant colony optimization and beam search, which is an incomplete branch and bound derivative. Our results show that Beam-ACO is able to find new best solutions for 31 out of 60 benchmark instances that we chose for the experimental evaluation of the algorithm.
\end{abstract}

\section{INTRODUCTION}

The longest common subsequence (LCS) problem is one of the classical string problems. Given a problem instance $(S, \Sigma)$, where $S=\left\{s_{1}, s_{2}, \ldots, s_{n}\right\}$ is a set of $n$ strings over a finite alphabet $\Sigma$, the problem consists in finding a longest string $t^{*}$ that is a subsequence of all the strings in $S$. Such a string $t^{*}$ is called a longest common subsequence of the strings in $S$. Note that a string $t$ is called a subsequence of a string $s$, if $t$ can be produced from $s$ by deleting characters. For example, $d g a$ is a subsequence of adagtta. Traditional computer science applications of this problem are in data compression [19], syntactic pattern recognition [13], file comparison [1], text edition [15] and query optimization in databases [16]. More recent applications include computational biology [18], [11] and the production of circuits in field programmable gate arrays [6]. The LCS problem was shown to be NP-hard [14] for an arbitrary number $n$ of input strings.

Due to its classical nature, the LCS problem has attracted quite a lot of research efforts over the past decades. Much work has been dedicated to the development of efficient dynamic programming procedures (see, for example, [2]). The body of work on approximate methods is dominated by constructive one-pass heuristics [9], [10]. Moreover, metaheuristics have been proposed in [17], [8], [12]. In [3] we recently published the current state-of-the-art algorithm for the LCS problem. The results of this algorithm, which is based on beam search (BS), have shown that none of the earlier algorithms came even close to derive good solutions for difficult problem instances. In other words, BS has shown to be largely superior to any other existing technique for what concerns the LCS problem. In this work we try to improve over the state-of-the-art results of BS by adding a learning component to $\mathrm{BS}$. The resulting algorithm is a hybrid between the metaheuristic ant colony optimization (ACO) [7] and beam search. The interested reader might note that we already published a preliminary version of this

Christian Blum is with the ALBCOM Research Group, Universitat Politècnica de Catalunya, c/ Jordi Girona 1-3, Campus Nord, Omega 112, 08034 Barcelona, Spain (phone: +34 9341 37884; email: cblum@1si.upc.edu).
Beam-ACO approach in [5]. However, the purpose of [5] was rather didactical in the sense that we aimed at showing the utility of hybridizing ant colony optimization with beam search. The LCS problem exclusively served as an example. In contrast, the algorithm that we present in this work, which is a further development of the one proposed in the above mentioned paper, aims at high performance. As we will show in the section on experimental results our algorithm is able to find new best solutions for 31 of the 60 problem instances that we chose for testing.

The organization of this paper is as follows. In Section II we present the proposed Beam-ACO approach. In Section III we outline the experimental evaluation. Finally, in Section IV we offer conclusions and an outlook to future work.

\section{BEAM-ACO}

The specific ACO algorithm that we used for adding a learning component to beam search is a standard $\mathcal{M A X}$ $\mathcal{M I N}$ Ant System implemented in the hyper-cube framework (HCF), see [4]. The pseudo-code of the algorithm is given in Algorithm 1. As usual, the data structures of the algorithm are: (1) the best-so-far solution $T^{b s}$, i.e., the best solution generated since the start of the algorithm; (2) the restart-best solution $T^{r b}$, that is, the best solution generated since the last restart of the algorithm; (3) the convergence factor $c f, 0 \leq c f \leq 1$, which is a measure of how far the algorithm is from convergence; and (4) the Boolean variable bs_update, which becomes true when the algorithm reaches convergence.

One of the crucial points of any ACO algorithm is the pheromone model $\mathcal{T}$. In the case of the LCS problem, the definition of the pheromone model is not a trivial task as, for example, in the case of the TSP. After some experimentation we decided to use a pheromone model $\mathcal{T}$ that contains for each position $j$ of a string $s_{i} \in S$ a pheromone value $0 \leq$ $\tau_{i j} \leq 1$, that is, $\mathcal{T}=\left\{\tau_{i j}|i=1, \ldots, n, j=1, \ldots,| s_{i} \mid\right\}$. Note that value $\tau_{i j} \in \mathcal{T}$ indicates the goodness of adding the letter at position $j$ of string $s_{i}$ to the solution under construction: the greater $\tau_{i j}$, the greater is the goodness of adding the corresponding letter. This pheromone model implies a specific way of representing solutions, henceforth called ACO-solutions. Note that any common subsequence $t$ of the strings in $S$ - that is, any solution $t$ - can be translated in a well-defined way into a unique ACO-solution $T=$ $\left\{T_{i j} \in\{0,1\}|i=1, \ldots, n, j=1, \ldots,| s_{i} \mid\right\}$. This is done as follows: for each string $s_{i} \in S$ we search the positions of the letters of $t$ in $s_{i}$ such that each letter is in its left-most position possible. Then, these positions $j$ are set to 1 in $T$, that is, $T_{i j}=1$, and $T_{i j}=0$ otherwise. For example, consider an instance $S=\{a c b c a d b b d, c a b d a c d c d, b a b c d d a a b\}$ and a 


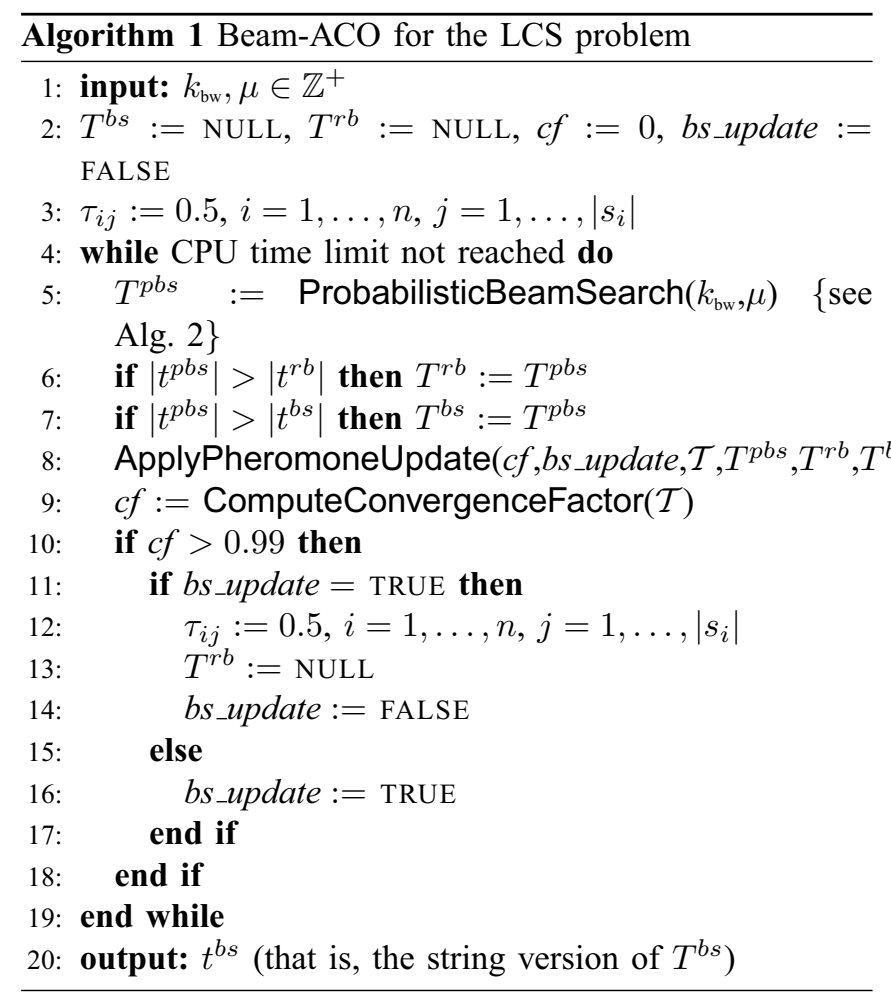

possible solution $t=a b c d d$. This solution translates into the ACO-solution $T=\{101101001,011001101,011111000\}$.

The algorithm works as follows. After the initialization of the pheromone values to 0.5 , each iteration consists of the following steps. First, beam search (BS) is applied in a probabilistic way, based on pheromone values. The working of BS is outlined further below in Section II-A. This generates a solution $T^{p b s}$. Second, the pheromone update is conducted by procedure ApplyPheromoneUpdate $(c f$, bs_update, $\left.\mathcal{T}, T^{p b s}, T^{r b}, T^{b s}\right)$. Third, a new value for the convergence factor $c f$ is computed. Depending on this value, as well as on the value of the Boolean variable bs_update, a decision on whether to restart the algorithm or not is made. If the algorithm is restarted, all the pheromone values are reset to their initial value (that is, 0.5). The algorithm is iterated until a maximum computation time limit is reached. Once terminated, the algorithm returns the string version $t^{b s}$ of the best-so-far ACO-solution $T^{b s}$, the best solution found. In the following we describe the two remaining procedures of Algorithm 1 in more detail.

ApplyPheromoneUpdate $\left(c f, b s \_u p d a t e, \mathcal{T}, T^{p b s}, T^{r b}, T^{b s}\right)$ : As usual in $\mathcal{M}$ MAS algorithms implemented in the HCF, three solutions are used for updating the pheromone values. These are the solution $T^{p b s}$ generated by beam search, the restart-best solution $T^{r b}$, and the best-so-far solution $T^{b s}$. The influence of each solution on the pheromone update depends on the state of convergence of the algorithm as measured by the convergence factor $c f$. Each pheromone value $\tau_{i j} \in \mathcal{T}$ is updated as follows:

$$
\tau_{i j}:=\tau_{i j}+\rho \cdot\left(\xi_{i j}-\tau_{i j}\right),
$$

where

$$
\xi_{i j}:=\kappa_{p b s} \cdot T_{i j}^{p b s}+\kappa_{r b} \cdot T_{i j}^{r b}+\kappa_{b s} \cdot T_{i j}^{b s},
$$

where $\kappa_{p b s}$ is the weight (that is, the influence) of solution $T^{p b s}, \kappa_{r b}$ is the weight of solution $T^{r b}, \kappa_{b s}$ is the weight of solution $T^{b s}$, and $\kappa_{p b s}+\kappa_{r b}+\kappa_{b s}=1$. After the pheromone update rule (Equation 1) is applied, pheromone values that exceed $\tau_{\max }=0.999$ are set back to $\tau_{\max }$ (similarly for $\left.\tau_{\min }=0.001\right)$. This is done in order to avoid a complete convergence of the algorithm, which is a situation that should be avoided. Equation 2 allows to choose how to weight the relative influence of the three solutions used for updating the pheromone values. For our application we used a standard update schedule as shown in Table I.

ComputeConvergenceFactor $(\mathcal{T})$ : The convergence factor $c f$, which is a function of the current pheromone values, is computed as follows:

$$
c f:=2\left(\left(\frac{\sum_{\tau_{i j} \in \mathcal{T}} \max \left\{\tau_{\max }-\tau_{i j}, \tau_{i j}-\tau_{\min }\right\}}{|\mathcal{T}| \cdot\left(\tau_{\max }-\tau_{\min }\right)}\right)-0.5\right)
$$

Note that when the algorithm is initialized (or reset) it holds that $c f=0$. On the other side, when the algorithm has converged, then $c f=1$. In all other cases, $c f$ has a value in $(0,1)$. This completes the description of the learning component of our Beam-ACO approach for the LCS problem.

\section{A. Beam Search}

In the following we give a technical description of the (probabilistic) BS algorithm that is used for construction solutions within the ACO framework. The implemented BS algorithm-see Algorithm 2-works roughly as follows. Solutions are constructed from left to right. Partial solutions are extended by appending exactly one letter. The algorithm requires two input parameters: $k_{\mathrm{bw}} \in \mathbb{Z}^{+}$is the so-called beam width and $\mu \in \mathbb{R}^{+} \geq 1$ is a parameter that is used to determine the number of children that can be chosen at each step. For each application the algorithm maintains a set $B$ of subsequences (that is, partial solutions) called the beam. At the start of the algorithm $B$ only contains the empty string denoted by $\emptyset$, that is, $B:=\emptyset$. Let $C$ denote the set of all possible extensions of the subsequences in $B$. At each step, $\left\lfloor\mu k_{\mathrm{bw}}\right\rfloor$ extensions from $C$ are chosen based on a greedy function and the pheromone values. In case a chosen extension represents a complete solution, it is stored in set $B_{\text {compl }}$. Otherwise, it is added to set $B$, in case its upper bound value, denoted by $\mathrm{UB}()$, is greater than the length of the best-so-far solution $t_{\text {bsf }}$. At the end of each step, the new beam $B$ is reduced if it contains more than $k_{\mathrm{bw}}$ partial solutions. This is done by evaluating the subsequences in $B$ by the above mentioned upper bound function $\operatorname{UB}()$, and by selecting the $k_{\mathrm{bw}}$ subsequences with the greatest upper bound values. In the following we explain the four different 
TABLE I

SETTING OF $\kappa_{p b s}, \kappa_{r b}, \kappa_{b s}$, AND $\rho$ DEPENDING ON THE CONVERGENCE FACTOR $c f$ AND THE BOOLEAN CONTROL VARIABLE $b s \_u p d a t e$

\begin{tabular}{|c|c|c|c|c|c|}
\hline & \multicolumn{4}{|c|}{ bs_update $=$ FALSE } & \multirow{2}{*}{$\begin{array}{l}\text { bs_update } \\
=\mathrm{TRUE}\end{array}$} \\
\hline & $c f<0.4$ & $c f \in[0.4,0.6)$ & $c f \in[0.6,0.8)$ & $c f \geq 0.8$ & \\
\hline$\kappa_{i b}$ & 1 & $2 / 3$ & $1 / 3$ & 0 & 0 \\
\hline$\kappa_{r b}$ & 0 & $1 / 3$ & $2 / 3$ & 1 & 0 \\
\hline$\kappa_{b s}$ & 0 & 0 & 0 & 0 & 1 \\
\hline$\rho$ & 0.2 & 0.2 & 0.2 & 0.15 & 0.15 \\
\hline
\end{tabular}

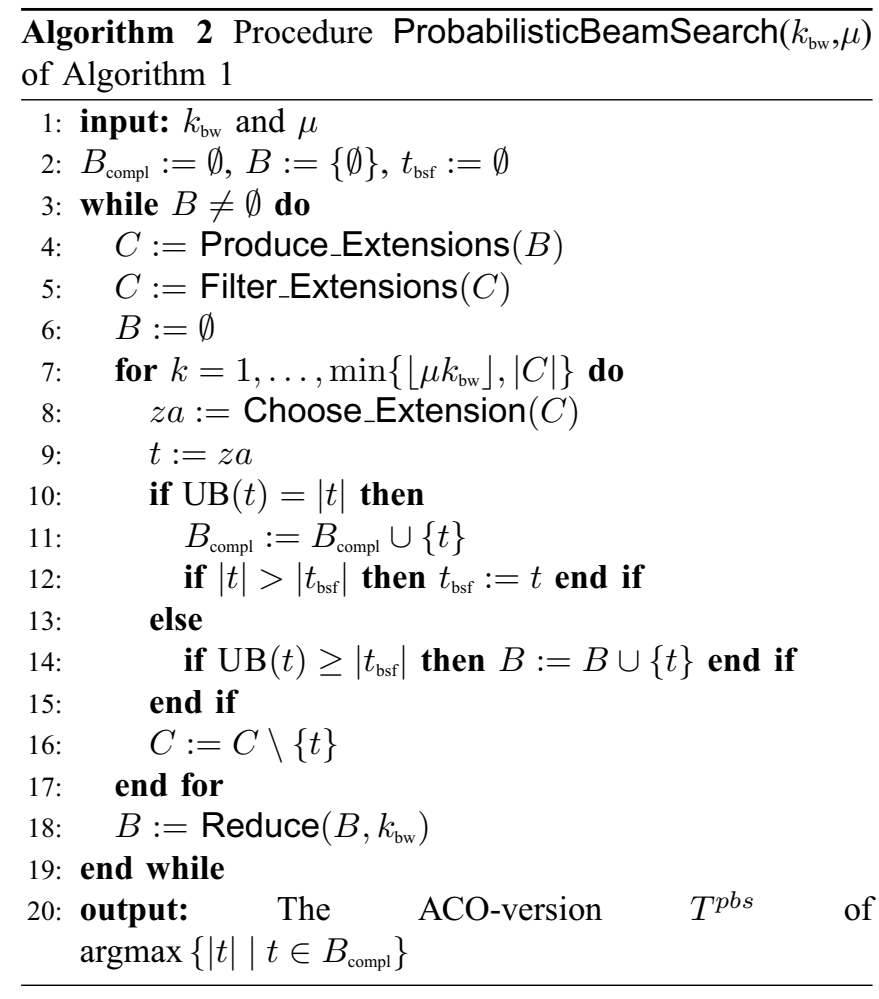

functions of Algorithm 2 in detail.

Produce_Extensions $(B)$ : Given the current beam $B$ as input, function Produce_Extensions $(B)$ produces the set $C$ of non-dominated extensions of all the subsequences in $B$. This is done as follows. Given a partial solution $t$ to a problem instance $(\mathcal{S}, \Sigma)$ :

1) Let $s_{i}=x_{i} \cdot y_{i}$ be the partition of $s_{i}$ into substrings $x_{i}$ and $y_{i}$ such that $t$ is a subsequence of $x_{i}$, and $y_{i}$ has maximal length. Given this partition, which is welldefined, we keep track of position pointers $p_{i}:=\left|x_{i}\right|$ for $i=1, \ldots, n$. The partition into substrings as well as the corresponding position pointers are shown by means of an example in Figure 1.

2) The position of the first appearance of a letter $a \in \Sigma$ in a string $s_{i} \in \mathcal{S}$ after the position pointer $p_{i}$ is welldefined and denoted by $p_{i}^{a}$. In case letter $a \in \Sigma$ does not appear in $y_{i}, p_{i}^{a}$ is set to $\infty$. Again, see Figure 1 for an example.
3) Letter $a \in \Sigma$ is called dominated, if there exists at least one letter $b \in \Sigma, a \neq b$, such that $p_{i}^{b}<p_{i}^{a}$ for $i=1, \ldots, n$. For an example consider the partial solution $t=b$ for the problem instance considered in Figure 1. As letter $a$ always appears before letter $d$ in $y_{i}(\forall i \in\{1,2,3\})$, we say that $a$ dominates $d$.

4) $\Sigma_{t}^{\text {nd }} \subseteq \Sigma$ denotes the set of non-dominated letters of the alphabet $\Sigma$ with respect to $t$. Obviously it is required that a letter $a \in \sum_{t}^{\text {nd }}$ appears in each string $s_{i}$ at least once after the position pointer $p_{i}$.

More specifically, $C$ is the set of subsequences $t a$, where $t \in B$ and $a \in \Sigma_{t}^{\text {nd }}$.

Filter_Extensions $(C)$ : This second function extends the non-domination relation - as defined above-from the extensions of one specific subsequence to the extensions of different subsequences of the same length. Formally, given two extensions $t a, z b \in C$, where $t \neq z$ but not necessarily $a \neq b, t a$ dominates $z b$ if and only if the position pointers concerning $a$ appear before the position pointers concerning $b$ in the corresponding remaining parts of the $n$ strings. All dominated partial solutions are removed from $C$.

Choose_Extension $(C)$ : The choice of an extension from $C$ is done as follows. First, based on the values of a greedy function and the pheromone values a probability for each extensions $t a \in C$ is generated. More specifically, the greedy function of an extension $t a \in C$ is the following one:

$$
\eta(t a):=\left(\sum_{i=1, \ldots, n} \frac{p_{i}^{a}-p_{i}}{\left|y_{i}\right|}\right)^{-1}
$$

However, instead of using directly these greedy values, we decided to use the corresponding ranks instead. For evaluating a child $v=t a \in C$ we use the sum of the ranks of the greedy weights that correspond to the construction steps performed to construct string $v$. Let us assume that $v$ is on the $i$-th level of the search tree, and let us denote the sequence of characters that forms string $v$ by $v_{1} \ldots v_{i}$, that is, $v=v_{1} \ldots v_{i}$. Then,

$$
\nu(v):=\sum_{j=1}^{i} r\left(v_{1} \ldots v_{j}\right),
$$

where $v_{1} \ldots v_{j}$ denotes the substring of $v$ from position 1 to position $j$, and $r\left(v_{1} \ldots v_{j}\right)$ denotes the rank of the corre- 


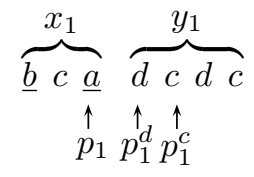

(a) String $s_{1}$

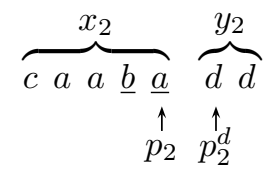

(b) String $s_{2}$

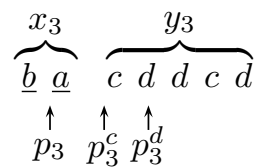

(c) String $s_{3}$

Fig. 1. In this graphic we consider instance $I^{\mathrm{ex}}=\left(\mathcal{S}=\left\{s_{1}, s_{2}, s_{3}\right\}, \Sigma=\{a, b, c, d\}\right)$, where $s_{1}=b c a d c d c, s_{2}=c a a b a d d$, and $s_{3}=b a c d d c d$. Moreover, the current partial solution is $t=b a$. Figures (a), (b), and (c) show the corresponding division of $s_{i}$ into $x_{i}$ and $y_{i}$, as well as the setting of the position pointers $p_{i}$ and the next positions of the 4 letters in $y_{i}$. In case a letter does not appear in $y_{i}$, the corresponding pointer is set to $\infty$. This is the case for letters $a$ and $b$ in $y_{1}: p_{1}^{a}:=\infty$ and $p_{1}^{b}:=\infty$.

sponding greedy value. With this definition, the probability for each $v=t a \in C$ is computed as follows:

$$
\mathbf{p}(v=t a \mid C)=\frac{\left(\min _{i=1, \ldots, n}\left\{\tau_{i p_{i}^{a}}\right\} \cdot \nu(v)^{-1}\right)}{\sum_{w=z b \in C}\left(\min _{i=1, \ldots, n}\left\{\tau_{i p_{i}^{b}}\right\} \cdot \nu(w)^{-1}\right)}
$$

Remember in this context, that $p_{i}^{a}$ was defined as the next position of letter $a$ after position pointer $p_{i}$ in string $s_{i}$. The intuition of choosing the minimum of the pheromone values corresponding to the next positions of a letter in the $n$ given strings is as follows: If at least one of these pheromone values is low, the corresponding letter should not yet be appended to the string, because there is another letter that should be appended first. Finally, each application of function Choose_Extension $(C)$ is either executed probabilistically, or deterministically. This is decided with uniform probability. In case of a deterministic choice, we simply choose the extension with the highest probability value. The probability for a deterministic choice, also called the determinism rate, is henceforth denoted by $q \in[0,1]$.

$\operatorname{Reduce}\left(B, k_{\mathrm{bw}}\right)$ : In case $|B|>k_{\mathrm{bw}}$, this function removes from $B$ step-by-step those subsequences $t$ that have an upper bound value $\mathrm{UB}(t)$ smaller or equal to the upper bound value of all the other subsequences in $B$. The removal process stops once $|B|=k_{\mathrm{bw}}$. For the explanation of the upper bound function that we used remember that a given common subsequence $t$ splits each string $s_{i} \in \mathcal{S}$ into a first part $x_{i}$ and into a second part $y_{i}$, that is, $s_{i}=x_{i} \cdot y_{i}$. Henceforth, $\left|y_{i}\right|_{a}$ denotes the number of occurrences of letter $a \in \Sigma$ in $y_{i}$. The upper bound value of $t$ is defined as follows:

$$
\mathrm{UB}(t):=|t|+\sum_{a \in \Sigma} \min \left\{\left|y_{i}\right|_{a} \mid i=1, \ldots, n\right\}
$$

In words, for each letter $a \in \Sigma$ the minimum number (over $i=1, \ldots, n)$ of its occurrences in $y_{i}$ is taken. Summing up these minima and adding the resulting sum to the length of $t$ results in the upper bound value. Note that this upper bound function can be efficiently computed by keeping appropriate data structures.

\section{EXPERIMENTAL EVALUATION}

We implemented Beam-ACO in ANSI C++ using GCC 3.2.2 for compiling the software. The experimental results that we outline in the following were obtained on a PC with an AMD64X2 4400 processor and 4 Gigabyte of memory. The set of benchmark instances that we chose for testing was introduced by Shyu and Tsai in [17]. Their instances are biologically inspired, and thus, they consider alphabets of size $|\Sigma|=4$, corresponding to DNA sequences, and of size $|\Sigma|=20$, corresponding to protein sequences. Shyu and Tsai provided three different types of instances. One is randomly generated and we denote this set henceforth as Random. The other two sets consist of real DNA and protein sequences of rats and viruses, Rat respectively Virus. Each of these three sets consists of 20 instances, 10 with an alphabet size of 4 and another 10 with an alphabet size of 20. The string length is always 600 , while the number of strings per instance varies from 10 to 200 , that is $n \in\{10,15,20,25,40,60,80,100,150,200\}$.

First we conducted tuning experiments. We considered the following three parameters for tuning: (1) The beam width $k_{\text {bw }} \in\{20,30,40\}$, (2) parameter $\mu \in\{1.5,2.0,3.0\}$, and (3) the determinism rate $q \in\{0,0.2,0.4,0.6,0.7,0.8,0.9\}$. For tuning we used the 20 instances from the Virus set. We applied Beam-ACO for each combination of $k_{\mathrm{bw}}, \mu$ and $q 10$ times with a computation time limit of 200 seconds to each problem instance. The tuning results can be summarized as follows. The beam width has no significant influence on both the quality and the running time of the algorithm. As the setting with $k_{\mathrm{bw}}=40$ seemed to work slightly better than the other settings, we decided to use this setting for the rest of the experiments. In contrast, both the determinism rate and the setting of parameter $\mu$ have a significant effect on the quality of the achieved results. Both is shown by means of graphics in Figure 2. Figures 2(a) and 2(b) show for each setting of the determinism rate $(q)$ the ranks of the average results achieved by the algorithm over a set of problem instances in form of box plots. For these experiments we adopted a setting of $\mu=2.0$. Figure 2(a) shows the box-plots for the 10 instances with $|\Sigma|=4$, while Figure 2(b) shows the same information for the 10 instances with $|\Sigma|=20$. Note that the lower a box the better the corresponding algorithm setting. From the results it is clear that a determinism rate of $q=0.0$ does not work well at all. This means that at least some degree of determinism is needed. While the results for the instances with $|\Sigma|=4$ are not really conclusive for what concerns the choice of $q$ from $\{0.2,0.4,0.6,0.7,0.8,0.9\}$, the results for the instances with $|\Sigma|=20$ clearly show that the 
algorithm's performance keeps increasing with increasing determinism rate until $q=0.8$. Therefore, we decided to adopt this setting for all further experiments. The graphics in Figures 2(c) and 2(d) show the results of Beam-ACO for the three different settings of $\mu$. Note that for these experiments $k_{\text {bw }}$ was fixed to 40 and $q$ to 0.8 . The curves show the evolution of the algorithm rank that was computed with respect to the average algorithm performance over 10 trials for the 10 instances with $|\Sigma|=4$, respectively $|\Sigma|=20$. This time the results for the instances with $|\Sigma|=4$ are more conclusive. While for small instances-with respect to the number of strings - a rather small setting of $\mu$ seems required, larger instances seem to require a larger $\mu$. In particular, for $n \in\{10,15\}$ the setting $\mu=1.5$ is best, for $n \in\{20,25,40,60\}$ the setting $\mu=2.0$ is best, while for $n \in\{80,100,150,200\}$ the setting of $\mu=3.0$ is best. In fact, these are the settings that we have adopted for the final experiments for what concerns instances with $|\Sigma|=4$ from sets Rat and Random. Concerning the instances with $|\Sigma|=20$, the tuning results are less conclusive (see Figure 2(d)). Therefore, we decided for a reasonable compromise achieved by the setting $\mu=2.0$ for all remaining experiments concerning instances with $|\Sigma|=20$.

With the settings as described above we applied BeamACO for 10 times with a computation time limit of 200 seconds to each of the 60 problem instances. The final results - separated for the three instance sets-are presented in Tables II, III and IV. All three tables provide information about the results of three different algorithms. Apart from the results of Beam-ACO we also present the results of the standard ACO algorithm proposed by Shyu and Tsai in [17] and the results of beam search [3], which is the current stateof-the-art algorithm. For ACO we provide for each instance the information that was given in [17], that is, the average results and the average computation times over 10 runs, as well as the corresponding standard deviations. For beam search, which is a deterministic heuristic, we provide the result in addition to the computation time. For Beam-ACO we provide the same information as for ACO. In addition, the first column concerning Beam-ACO contains the best result achieved for each instance over 10 runs. In case of a gray background, Beam-ACO has achieved a new best solution for the corresponding instance. On the other side, when BeamACO was not able to reach the performance of beam search, the corresponding result of beam search is printed in italic style.

The following observations can be made. Altogether, Beam-ACO is able to produce new best solutions in 31 out of 60 cases. Only in 7 cases, Beam-ACO produces a result that is inferior to the one of beam search. Most of the new best solutions are obtained for instances with $|\Sigma|=4$. On the other side, all cases in which Beam-ACO does not reach the performance of beam search concern instances with $|\Sigma|=20$. In our opinion, this indicates that beam search is working better for instances with larger alphabets, while for instances with smaller alphabets there was still room for improvement. Concerning the computation times, the processor that we used for evaluating Beam-ACO is presumably more than twice as fast than the processors used for the evaluation of $\mathrm{ACO}$ and beam search. This means that Beam-ACO is significantly slower than ACO and beam search. However, as the LCS problem is not a time critical problem, it is much more important to improve in terms of solution quality, even with the disadvantage of increased running times. A critical reader might remark at this point that the performance of beam search may be increased by choosing a larger beam width. Therefore, we applied beam search (as published in [3]) to all 60 problem instances with an increased beam width of 500 . Note that the original setting in [3] was 100. The results that are presented in Table V show indeed that the performance of beam search increases. However, this increase in quality comes at the cost of a considerably elevated computation time. New best solutions are found in 17 cases (as marked by a gray background). However, in 16 cases (especially for instances with $|\Sigma|=4$ and a rather small number of strings) beam search with the setting of $k_{\mathrm{bw}}=500$ is inferior to Beam-ACO. These cases are marked by an asterisk. This demonstrates clearly the utility of adding a learning component to beam search as done in Beam-ACO.

\section{CONCLUSIONS}

In this work we have proposed an enhanced Beam-ACO approach for the longest common subsequence problem. The computational results, obtained on 60 problem instances from the literature, have shown that Beam-ACO is able to find new best solutions in 31 cases. In general, the results indicate the usefulness of adding a learning component in the style of Beam-ACO to beam search.

Future work will be concerned with the development of an effective local search algorithm for enhancing Beam-ACO. This is a challenging task, because at first sight the longest common subsequence problem does not seem suitable for the development of local search algorithms.

\section{ACKNOWLEDGEMENTS}

This work was supported by grant TIN2007-66523 (FORMALISM) of the Spanish government. Moreover, Christian Blum acknowledges support from the Ramón y Cajal program of the Spanish Ministry of Science and Innovation.

\section{REFERENCES}

[1] A. Aho, J. Hopcroft, and J. Ullman. Data structures and algorithms. Addison-Wesley, Reading, MA, 1983.

[2] L. Bergroth, H. Hakonen, and T. Raita. A survey of longest common subsequence algorithms. In Proceedings of SPIRE 2000 - 7th International Symposium on String Processing and Information Retrieval, pages 39-48. IEEE press, 2000.

[3] C. Blum, M. J. Blesa, and M. López Ibáñez. Beam search for the longest common subsequence problem. Computers \& Operations Research, 36(12):3178-3186, 2009.

[4] C. Blum and M. Dorigo. The hyper-cube framework for ant colony optimization. IEEE Transactions on Man, Systems and Cybernetics Part B, 34(2):1161-1172, 2004. 


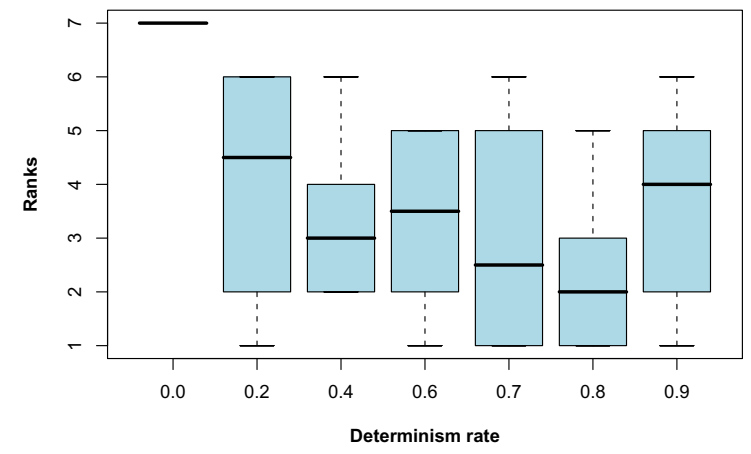

(a) Virus instances with $|\Sigma|=4$

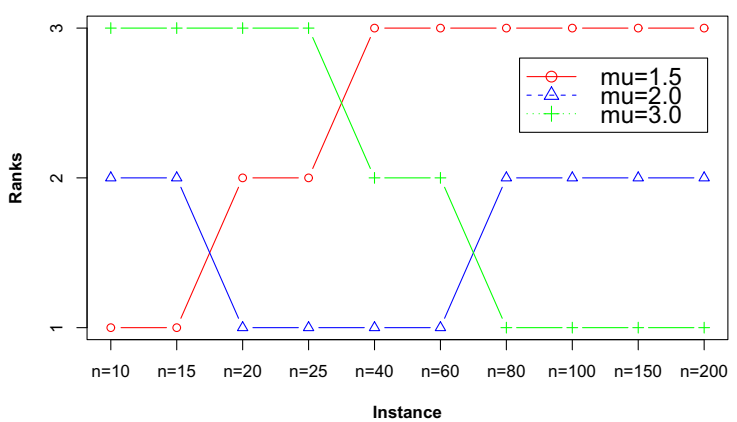

(c) Virus instances with $|\Sigma|=4$

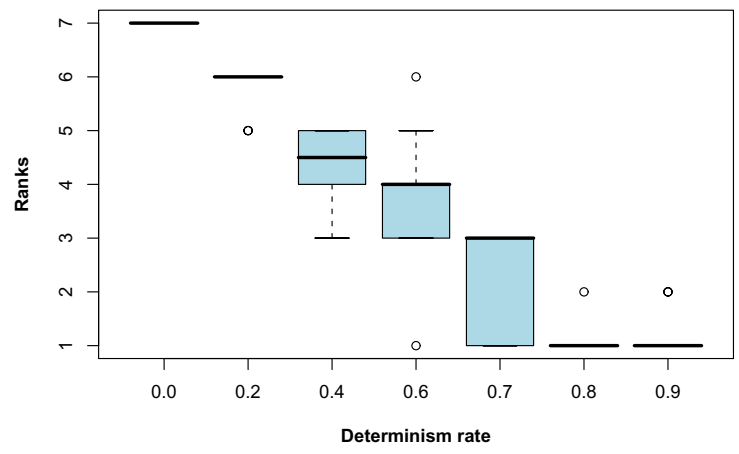

(b) Virus instances with $|\Sigma|=20$

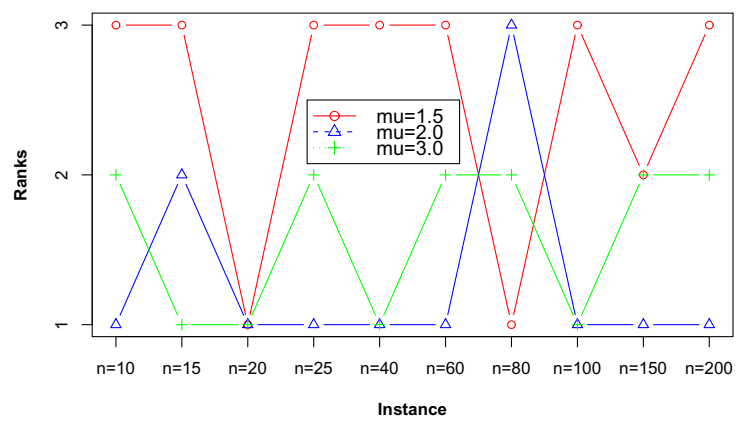

(d) Virus instances with $|\Sigma|=20$

Fig. 2. Tuning results presented in graphical form. Figures (a) and (b) concern the tuning of the determinism rate, whereas Figures (c) and (d) present results concerning the tuning of parameter $\mu$. More information is given in the text.

[5] C. Blum and M. Mastrolilli. Using branch \& bound concepts in construction-based metaheuristics: Exploiting the dual problem knowledge. In T. Bartz-Beielstein et al., editor, Proceedings of HM 2007 4th International Workshop on Hybrid Metaheuristics, volume 4771 of Lecture Notes in Computer Science, pages 123-139. Springer Verag, Berlin, Germany, 2007.

[6] P. Brisk, A. Kaplan, and M. Sarrafzadeh. Area-efficient instruction set synthesis for reconfigurable system-on-chip design. In Proceedings of the 4lst Design Automation Conference, pages 395-400. IEEE press, 2004.

[7] M. Dorigo and T. Stützle. Ant Colony Optimization. MIT Press, Cambridge, MA, 2004

[8] T. Easton and A. Singireddy. A large neighborhood search heuristic for the longest common subsequence problem. Journal of Heuristics, 14(3):271-283, 2008

[9] C. B. Fraser. Subsequences and supersequences of strings. $\mathrm{PhD}$ thesis, University of Glasgow, 1995.

[10] K. Huang, C. Yang, and K. Tseng. Fast algorithms for finding the common subsequences of multiple sequences. In Proceedings of the International Computer Symposium, pages 1006-1011. IEEE press, 2004.

[11] T. Jiang, G. Lin, B. Ma, and K. Zhang. A general edit distance between RNA structures. Journal of Computational Biology, 9(2):371-388, 2002

[12] B. A. Julstrom and B. Hinkemeyer. Starting from scratch: Growing longest common subsequences with evolution. In T. P. Runarsson, H.-G. Beyer, E. K. Burke, J. J. Merelo Guervós, L. D. Whitley, and X. Yao, editors, Proceedings of PPSN 2006 - 9th International Conference on Parallel Problem Solving from Nature, volume 4193 of Lecture Notes in Computer Science, pages 930-938. Springer Verag, Berlin, Germany, 2006.

[13] S. Y. Lu and K. S. Fu. A sentence-to-sentence clustering procedure for pattern analysis. IEEE Transactions on Systems, Man and Cybernetics, 8(5):381-389, 1978

[14] D. Maier. The complexity of some problems on subsequences and supersequences. Journal of the ACM, 25:322-336, 1978.

[15] D. Sankoff and J. B. Kruskal. Time Warps, String Edits, and Macromolecules: The Theory and Practice of Sequence Comparison. Addison-Wesley, Reading, UK, 1983.

[16] T. Sellis. Multiple query optimization. ACM Transactions on Database Systems, 13(1):23-52, 1988.

[17] S. J. Shyu and C.-Y. Tsai. Finding the longest common subsequence for multiple biological sequences by ant colony optimization. Computers \& Operations Research, 36(1):73-91, 2009.

[18] T. Smith and M. Waterman. Identification of common molecular subsequences. Journal of Molecular Biology, 147(1):195-197, 1981.

[19] J. Storer. Data Compression: Methods and Theory. Computer Science Press, MD, 1988. 
TABLE II

RESULTS FOR THE RANDOM INSTANCES.

\begin{tabular}{|c|c|c|c|c|c|c|c|c|c|c|c|c|}
\hline \multicolumn{2}{|c|}{ Instance } & \multirow[b]{2}{*}{ avg } & \multicolumn{3}{|c|}{ ACO } & \multicolumn{2}{|c|}{ Beam search } & \multicolumn{5}{|c|}{ Beam-ACO } \\
\hline$|\Sigma|$ & $n$ & & std & time (s) & std & result & time (s) & best & avg & std & time & std \\
\hline \multirow[t]{10}{*}{4} & 10 & 197.2 & (2.0) & 10.7 & $(2.0)$ & 211 & 9.8 & 216 & 215.70 & $(0.48)$ & 97.56 & (39.98) \\
\hline & 15 & 185.2 & (1.3) & 15.7 & (5.4) & 194 & 13.2 & 197 & 196.60 & $(0.52)$ & 79.90 & $(68.80)$ \\
\hline & 20 & 176.2 & (1.3) & 11.4 & $(0.8)$ & 184 & 14.9 & 186 & 185.60 & $(0.52)$ & 134.70 & $(60.47)$ \\
\hline & 25 & 172.2 & $(0.7)$ & 15.4 & (1.7) & 179 & 15.8 & 181 & 179.90 & $(0.57)$ & 40.55 & $(25.88)$ \\
\hline & 40 & 161.4 & (1.3) & 23.8 & (10.3) & 167 & 21.0 & 170 & 169.30 & $(0.67)$ & 62.36 & (44.12) \\
\hline & 60 & 155.4 & (1.3) & 24.7 & (3.2) & 161 & 27.6 & 162 & 161.30 & $(0.48)$ & 53.07 & (71.78) \\
\hline & 80 & 151.6 & $(0.8)$ & 32.5 & $(5.9)$ & 156 & 33.5 & 156 & 155.90 & $(0.32)$ & 62.62 & (43.49) \\
\hline & 100 & 148.8 & (1.3) & 43.6 & (10.4) & 154 & 40.3 & 154 & 153.20 & $(0.42)$ & 46.06 & $(53.84)$ \\
\hline & 150 & 143.4 & $(0.8)$ & 57.2 & $(17.1)$ & 148 & 56.4 & 149 & 148.20 & $(0.42)$ & 35.64 & (18.68) \\
\hline & 200 & 141.0 & $(0.6)$ & 59.1 & (9.6) & 146 & 74.3 & 146 & 145.30 & $(0.48)$ & 57.36 & (23.94) \\
\hline \multirow[t]{10}{*}{20} & 10 & 54.0 & (1.1) & 7.4 & $(2.1)$ & 61 & 33.3 & 60 & 60.00 & $(0.00)$ & 6.32 & $(0.27)$ \\
\hline & 15 & 46.2 & (1.6) & 9.3 & $(2.5)$ & 51 & 37.6 & 51 & 50.70 & $(0.48)$ & 77.92 & $(68.24)$ \\
\hline & 20 & 42.4 & (1.3) & 11.4 & (4.9) & 47 & 39.5 & 45 & 45.00 & $(0.00)$ & 4.25 & $(0.13)$ \\
\hline & 25 & 40.0 & (1.1) & 10.5 & (2.3) & 43 & 39.5 & 43 & 43.00 & $(0.00)$ & 6.34 & $(0.29)$ \\
\hline & 40 & 34.2 & $(0.7)$ & 14.1 & (4.8) & 37 & 43.2 & 38 & 38.00 & $(0.00)$ & 6.80 & $(0.05)$ \\
\hline & 60 & 30.6 & $(0.8)$ & 17.3 & (1.3) & 34 & 46.5 & 34 & 34.00 & $(0.00)$ & 7.13 & $(0.06)$ \\
\hline & 80 & 29.0 & (1.1) & 22.9 & (3.0) & 32 & 53.2 & 32 & 32.00 & $(0.00)$ & 5.30 & $(0.14)$ \\
\hline & 100 & 28.4 & $(0.8)$ & 25.6 & $(0.1)$ & 31 & 59.2 & 31 & 31.00 & $(0.00)$ & 9.95 & (3.44) \\
\hline & 150 & 26.0 & $(0.4)$ & 40.8 & (7.4) & 29 & 75.6 & 29 & 29.00 & $(0.00)$ & 59.00 & (44.28) \\
\hline & 200 & 25.0 & $(0.2)$ & 55.4 & (4.7) & 27 & 98.0 & 27 & 27.00 & $(0.00)$ & 14.66 & $(4.20)$ \\
\hline
\end{tabular}

TABLE III

RESUltS FOR THE RAT INSTANCES

\begin{tabular}{cl|llll|ll|lllll}
\hline \multicolumn{2}{c|}{$\begin{array}{l}\text { Instance } \\
|\Sigma|\end{array}$} & \multicolumn{4}{c}{ ACO } & avg & std & time (s) & std & Beam search & \multicolumn{5}{c}{ Beam-ACO } \\
\hline 4 & 10 & 182.0 & $(2.4)$ & 7.4 & $(1.9)$ & 191 & 9.7 & 197 & 195.00 & $(1.33)$ & 74.13 & $(49.04)$ \\
& 15 & 166.6 & $(1.3)$ & 10.5 & $(2.4)$ & 173 & 12.3 & 177 & 176.80 & $(0.42)$ & 53.05 & $(54.11)$ \\
& 20 & 160.0 & $(1.3)$ & 12.5 & $(3.8)$ & 163 & 12.6 & 168 & 167.40 & $(0.52)$ & 24.28 & $(25.29)$ \\
& 25 & 155.8 & $(1.3)$ & 15.9 & $(4.0)$ & 162 & 15.8 & 164 & 163.20 & $(0.79)$ & 83.64 & $(66.10)$ \\
& 40 & 143.4 & $(0.8)$ & 21.0 & $(4.6)$ & 146 & 19.4 & 152 & 150.70 & $(1.06)$ & 105.43 & $(71.68)$ \\
& 60 & 142.4 & $(1.7)$ & 26.2 & $(8.9)$ & 144 & 26.7 & 147 & 146.60 & $(0.52)$ & 99.56 & $(66.72)$ \\
& 80 & 128.8 & $(0.7)$ & 29.9 & $(4.9)$ & 135 & 31.8 & 135 & 133.90 & $(0.88)$ & 78.32 & $(69.21)$ \\
& 100 & 124.6 & $(2.0)$ & 48.8 & $(17.9)$ & 132 & 38.5 & 134 & 133.10 & $(0.57)$ & 112.71 & $(44.70)$ \\
& 150 & 115.6 & $(1.3)$ & 35.0 & $(6.8)$ & 121 & 51.1 & 123 & 122.10 & $(0.32)$ & 65.98 & $(27.71)$ \\
& 200 & 114.6 & $(2.3)$ & 65.5 & $(14.0)$ & 121 & 69.1 & 121 & 119.80 & $(0.63)$ & 105.55 & $(48.99)$ \\
\hline 20 & 10 & 63.4 & $(1.3)$ & 9.2 & $(2.5)$ & 69 & 27.4 & 70 & 69.80 & $(0.42)$ & 84.63 & $(56.68)$ \\
& 15 & 56.6 & $(0.8)$ & 8.9 & $(2.4)$ & 60 & 36.7 & 61 & 60.20 & $(0.42)$ & 53.43 & $(63.22)$ \\
& 20 & 47.8 & $(0.7)$ & 14.6 & $(5.8)$ & 51 & 34.4 & 53 & 52.40 & $(0.52)$ & 34.45 & $(57.05)$ \\
& 25 & 46.2 & $(1.3)$ & 11.5 & $(1.2)$ & 51 & 39.0 & 51 & 50.50 & $(0.53)$ & 58.91 & $(61.80)$ \\
& 40 & 44.2 & $(1.3)$ & 14.6 & $(3.2)$ & 49 & 47.4 & 48 & 48.00 & $(0.00)$ & 12.25 & $(5.30)$ \\
& 60 & 43.0 & $(0.4)$ & 31.5 & $(6.9)$ & 46 & 60.3 & 45 & 45.00 & $(0.00)$ & 25.95 & $(25.96)$ \\
& 80 & 39.6 & $(0.8)$ & 32.4 & $(10.1)$ & 43 & 64.4 & 42 & 42.00 & $(0.00)$ & 20.52 & $(10.84)$ \\
& 100 & 37.0 & $(1.1)$ & 42.4 & $(8.8)$ & 38 & 64.8 & 37 & 37.00 & $(0.00)$ & 12.89 & $(6.32)$ \\
& 150 & 34.0 & $(1.1)$ & 49.1 & $(8.1)$ & 36 & 77.8 & 36 & 35.10 & $(0.32)$ & 15.35 & $(25.45)$ \\
& 200 & 32.4 & $(1.3)$ & 75.7 & $(17.0)$ & 33 & 101.0 & 33 & 32.10 & $(0.32)$ & 37.77 & $(25.14)$ \\
\hline
\end{tabular}


TABLE IV

RESULTS FOR THE VIRUS INSTANCES.

\begin{tabular}{|c|c|c|c|c|c|c|c|c|c|c|c|c|}
\hline \multicolumn{2}{|c|}{ Instance } & \multicolumn{4}{|c|}{ ACO } & \multicolumn{2}{|c|}{ Beam search } & \multicolumn{5}{|c|}{ Beam-ACO } \\
\hline$|\Sigma|$ & $n$ & avg & std & time (s) & std & result & time (s) & best & avg & std & time & std \\
\hline \multirow[t]{10}{*}{4} & 10 & 197.6 & (1.3) & 3.7 & $(0.7)$ & 212 & 11.6 & 217 & 216.00 & $(0.47)$ & 76.67 & $(51.96)$ \\
\hline & 15 & 183.6 & (1.3) & 7.9 & $(2.0)$ & 193 & 15.4 & 200 & 199.10 & $(0.32)$ & 64.56 & $(50.53)$ \\
\hline & 20 & 173.8 & (2.5) & 20.4 & (6.5) & 181 & 17.2 & 184 & 183.00 & $(0.67)$ & 49.84 & $(40.51)$ \\
\hline & 25 & 179.0 & (1.8) & 18.3 & (5.3) & 185 & 17.9 & 189 & 187.90 & $(0.74)$ & 98.31 & $(67.51)$ \\
\hline & 40 & 155.0 & (2.1) & 20.5 & (3.4) & 162 & 21.9 & 166 & 162.80 & (1.62) & 93.51 & $(56.30)$ \\
\hline & 60 & 150.6 & (1.3) & 30.8 & (9.3) & 158 & 29.1 & 160 & 158.60 & $(0.70)$ & 73.50 & $(47.35)$ \\
\hline & 80 & 145.8 & (1.3) & 45.5 & (6.9) & 153 & 36.0 & 154 & 153.30 & $(0.67)$ & 84.78 & $(59.96)$ \\
\hline & 100 & 143.4 & (2.7) & 23.8 & (10.3) & 150 & 43.9 & 151 & 150.00 & $(0.82)$ & 89.55 & $(61.29)$ \\
\hline & 150 & 141.6 & $(0.8)$ & 50.0 & (21.3) & 148 & 64.5 & 149 & 147.70 & $(0.95)$ & 91.72 & $(51.28)$ \\
\hline & 200 & 140.6 & (1.3) & 65.6 & (15.6) & 145 & 84.5 & 148 & 146.50 & (1.08) & 116.19 & (61.79) \\
\hline \multirow[t]{10}{*}{20} & 10 & 65.6 & $(0.8)$ & 3.5 & $(1.2)$ & 75 & 27.2 & 75 & 75.00 & $(0.00)$ & 10.31 & $(10.76)$ \\
\hline & 15 & 55.8 & (1.3) & 10.4 & (1.6) & 63 & 38.6 & 61 & 61.00 & $(0.00)$ & 33.16 & (20.35) \\
\hline & 20 & 53.6 & (1.3) & 10.8 & $(0.5)$ & 57 & 40.3 & 57 & 57.00 & $(0.00)$ & 33.98 & (24.81) \\
\hline & 25 & 49.6 & $(0.8)$ & 13.2 & (4.5) & 53 & 38.9 & 54 & 53.90 & $(0.32)$ & 51.61 & (45.97) \\
\hline & 40 & 46.4 & $(0.8)$ & 17.1 & (2.6) & 49 & 48.4 & 49 & 49.00 & $(0.00)$ & 16.91 & (10.71) \\
\hline & 60 & 43.4 & $(0.8)$ & 27.7 & $(4.2)$ & 45 & 56.1 & 47 & 47.00 & $(0.00)$ & 32.96 & (32.49) \\
\hline & 80 & 43.0 & $(0.4)$ & 38.1 & (11.4) & 44 & 67.4 & 44 & 44.00 & $(0.00)$ & 26.29 & (16.47) \\
\hline & 100 & 42.0 & (1.1) & 23.4 & (5.1) & 43 & 74.2 & 43 & 43.00 & $(0.00)$ & 76.22 & $(60.76)$ \\
\hline & 150 & 42.6 & $(0.8)$ & 71.4 & $(19.8)$ & 44 & 108.0 & 44 & 43.10 & $(0.32)$ & 26.49 & $(36.25)$ \\
\hline & 200 & 41.0 & $(0.2)$ & 78.9 & (21.7) & 43 & 140.0 & 43 & 43.00 & $(0.00)$ & 29.00 & (16.57) \\
\hline
\end{tabular}

TABLE V

RESULTS FOR BEAM SEARCH (PUBLISHED IN [3]) WITH AN INCREASED BEAM WIDTH OF 500 , THAT IS, $k_{\mathrm{BW}}=500$.

\begin{tabular}{|c|c|c|c|c|c|c|c|c|c|c|c|c|}
\hline \multirow[b]{3}{*}{$n$} & \multicolumn{4}{|c|}{ Random } & \multicolumn{4}{|c|}{ Rat } & \multicolumn{4}{|c|}{ Virus } \\
\hline & \multicolumn{2}{|c|}{$|\Sigma|=4$} & \multicolumn{2}{|c|}{$|\Sigma|=20$} & \multicolumn{2}{|c|}{$|\Sigma|=4$} & \multicolumn{2}{|c|}{$|\Sigma|=20$} & \multicolumn{2}{|c|}{$|\Sigma|=4$} & \multicolumn{2}{|c|}{$|\Sigma|=20$} \\
\hline & result & time (s) & result & time (s) & result & time (s) & result & time (s) & result & time (s) & result & time (s) \\
\hline 10 & *213 & 147 & 61 & 601 & ${ }^{*} 196$ & 166 & 70 & 297 & $* 215$ & 171 & 75 & 217 \\
\hline 15 & 200 & 219 & 52 & 929 & ${ }^{*} 176$ & 224 & $* 60$ & 419 & *196 & 216 & 63 & 333 \\
\hline 20 & 188 & 249 & 47 & 1120 & $* 165$ & 236 & 53 & 444 & ${ }^{*} 183$ & 249 & 58 & 564 \\
\hline 25 & 181 & 249 & 43 & 1060 & 164 & 252 & 51 & 523 & ${ }^{*} 184$ & 261 & 54 & 516 \\
\hline 40 & *169 & 294 & 38 & 955 & *148 & 284 & 48 & 677 & *163 & 299 & 49 & 612 \\
\hline 60 & 162 & 295 & 34 & 858 & 147 & 350 & 46 & 771 & 160 & 302 & $* 46$ & 583 \\
\hline 80 & 157 & 281 & 33 & 861 & 137 & 288 & 44 & 856 & 154 & 330 & 45 & 665 \\
\hline 100 & 155 & 314 & 31 & 849 & *133 & 323 & 39 & 676 & 152 & 316 & 43 & 629 \\
\hline 150 & *148 & 336 & 29 & 833 & 126 & 342 & 37 & 499 & $* 147$ & 360 & 45 & 794 \\
\hline 200 & 148 & 377 & 27 & 920 & 121 & 360 & 34 & 636 & 148 & 424 & 43 & 834 \\
\hline
\end{tabular}

